\title{
CONTROL OF A SURFACE OF DISCONTINUITY IN CONTINUOUS THICKNESS
}

\author{
N. G. BARTON, C.-H. LI and S. J. SPENCER ${ }^{1}$
}

(Received 15 March 1991; revised 18 April 1991)

\begin{abstract}
This paper examines the control of an interface between a suspension of sedimenting particles in liquid and a bed of dense-packed particles at the bottom of the suspension. The problem arises in the operation of continuous thickeners (e.g. in mineral processing) and is here mathematically described by a first order inhomogeneous partial differential equation for the concentration $C(x, t)$ of particles. The controlled variable is the height $H^{*}$ of the bed, and the control variables are the volume fluxes injected at the feed level and removed at the bed. A strategy to control the interface is devised, and control is confirmed and demonstrated by a series of numerical experiments.
\end{abstract}

\section{Introduction}

Sedimentation is a very old process for separating small particles from a liquid. Examples from antiquity include clarification of wine and beer (in which clear liquid is the goal) and primitive mineral processing (in which extraction of heavy sediments is the goal). In modern mineral processing, flotationin some senses the opposite process to sedimentation-is also exceedingly important.

Mathematically, sedimentation can be modelled by first-order partial differential equations which sometimes possess discontinuous solutions. These discontinuities are analogous to free boundaries in more common problems, involving change of phase or change of material properties, in which governing second-order partial differential equations are typical.

Our goal is to examine, in terms of a simple model, the existence and control of the most important discontinuity in sedimentation processes-that

${ }^{1}$ CSIRO Division of Mathematics and Statistics, P.O. Box 218, Lindfield, NSW Australia 2070.

(C) Copyright Australian Mathematical Society 1992, Serial-fee code 0334-2700/92 
between a suspension of particles and a high concentration zone at the bottom of the liquid. In many cases, this zone contains material at the maximum permissible concentration, and we therefore often used the term packed bed for it. The continuous thickener shown in Figure 1 is employed in our study. The thickener can be operated in two ways:

- Batch mode: Here, the inflow and outflow are both zero, and the initial suspension of particles is allowed to settle. A bed always forms in this case; at large times, the thickener has a packed bed at the bottom with clear fluid above it. Batch thickeners have been widely studied $[1,2,3,7,8,9,11,12,15,16,19]$.

- Continuous mode: Here, the inflow and outflow (and generally the overflow) are nonzero. This case is substantially more complicated than the batch case, and packed beds might or might not form depending on the manner in which the thickener is operated. Continuous thickeners have also been widely studied $[9,10,13,16]$.

There are many possibilities for modelling thickeners. We choose to use ideas due to Kynch [12] in which the principal assumption is that the sedimentation speed $v_{s}(C)$ of particles relative to the bulk velocity of fluid depends on the local concentration $C(x, t)$ of particles. Based on experimental results (see e.g. [15]) and the work of Kynch, we use the plausible functional representation (2) for $v_{s}(C)$. Emphasis on $v_{s}(C)$ as a given function is a considerable simplification that enables effects such as momentum associated with the sediment particles [7] and the precise nature of the bed [13] to be neglected. Our approach is both relatively simple and reasonably realistic for the applications envisaged.

As mentioned above, certain operating conditions of continuous thickeners lead to the formation of interfaces (discontinuities in concentration)

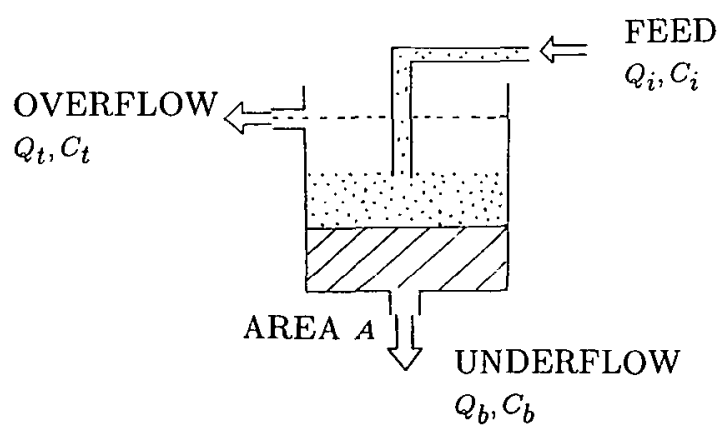

FIGURE 1. Schematic of a thickener. 
which rise or drop through the thickener. In particular, a high concentration zone sometimes forms and then either builds up or is pumped out of the thickener. In many cases, this zone has economic significance, and our goals are to determine operating conditions which control its depth, and to predict the concentration of material pumped off at the bottom. These operating conditions are deduced analytically (Section 3 ) and then confirmed and demonstrated numerically (Section 4,5 ).

The conclusions of the work are presented in Section 6. To highlight them:

- Control is possible through judicious choice of inflow and outflow pumping rates.

- Numerical schemes used to simulate thickener performance can be used as a basis of methods for control.

- The nature of the control depends sensitively on the $v_{s}(C)$ representation and on conditions at the boundary of the computational domain in $x-t$ space.

\section{Mathematical preliminaries}

The height of the thickener is $x=X$ and its cross-sectional area (assigned constant) is $A$. At height $x / X=h_{i}<1$, liquid containing identical particles is injected with volume flux $Q_{i}(t)=V_{i}(t) A$. The local fraction of the volume occupied by particles is denoted $C(x, t)$ (generally called the particle concentration or concentration). The concentration of the injected material is $C_{i}(t)$. At the bottom of the thickener, $x=0$, material is drawn off with volume flux $Q_{b}(t)=V_{b}(t) A$ and concentration $C_{b}(t)$. At height $x=X$, overflow of liquid takes place if $V_{i}>V_{b}$; if so, the overflow flux is $Q_{t}(t)=V_{t}(t) A$ with concentration $C_{t}(t)$.

The basic equation used in this work is one-dimensional conservation of particles

$$
\frac{\partial C}{\partial t}-\frac{\partial \Phi}{\partial x}=V_{i} C_{i} \delta\left(x-h_{i} X\right)
$$

where $\Phi$ is the (downward) volume flux of particles, $C$ the volume concentration, $t$ is time and $x$ is a coordinate measured vertically upwards. In practice, the injection nozzle is designed to minimise vertical fluid motions. Thus the one-dimensional model (1) should be a good approximation, particularly near the bottom of the thickener.

The sedimentation speed of particles (in a frame of reference moving at the bulk velocity of the mixture of particles and fluid) is denoted by $v_{s}(C)$. For this paper, we use the representation

$$
v_{s}(C)=v_{0}\left(1-C / C_{M}\right)^{q}+v_{1} C\left(C_{M}-C\right)
$$


in which $v_{0}$ is the sedimentation speed of an individual particle, $C_{M}$ is the maximum possible concentration of particles, $q$ is a positive constant and $v_{1}$ is the superficial velocity of a Darcy type flow of liquid. The first term in $v_{s}$ models free settling of particles as modified by the presence of other particles, and the second term is the dominant term for settling of a packed bed. This form for $v_{s}$ allows for a wide variety of behaviour which is compatible with sedimentation results in laboratory experiments and previous theoretical analyses (Auzerais et al. [1], Fryer \& Uhlherr [10], Kynch [12], Park et al. [15]).

The (downward) volume flux of particles is given by

$$
\Phi(C)=\phi(C)+V_{b} C-V_{i} C H\left(x-h_{i} X\right),
$$

in which $H\left(x-h_{i} X\right)$ is the Heaviside step function, the (downward) bulk velocity is

$$
V=V_{b}-V_{i} H\left(x-h_{i} X\right)
$$

and

$$
\phi(C)=v_{s}(C) C
$$

denotes (downward) sediment flux in a reference frame in which the bulk velocity is zero. Equations $(1,3,4)$ yield the basic equation

$$
\frac{\partial C}{\partial t}-\frac{\partial}{\partial x}\left[C\left(v_{s}+V_{b}-V_{i} H\left(x-h_{i} X\right)\right)\right]=V_{i} C_{i} \delta\left(x-h_{i} X\right), \quad(x, t) \in \Omega
$$

or equivalently

$$
\frac{\partial C}{\partial t}-\left(\gamma+V_{b}-V_{i} H\left(x-h_{i} X\right)\right) \frac{\partial C}{\partial x}=V_{i}\left(C_{i}-C\right) \delta\left(x-h_{i} X\right), \quad(x, t) \in \Omega
$$

in which $\Omega$ is the domain $0<x<X, t>0$,

$$
\gamma=\frac{d\left(C v_{s}(C)\right)}{d C}
$$

is the speed of propagation of density disturbances (with respect to stationary fluid), and the outflow and injection pumping speeds $V_{b}(t)$ and $V_{i}(t)$ can be assigned. The injected concentration $C_{i}(t)$ is also a suitable control variable, but is not used here for this purpose.

The solution of $(5,6)$ can be analysed using the method of characteristics (see e.g. [6]) and conditions need to be specified at those parts of the boundary $\partial \Omega$ at which characteristics enter $\Omega$. We assume initial data of the form $C(x, 0)=f_{0}(x)$ where

$$
f_{0}(x)=\left\{\begin{array}{l}
C_{1}, \quad \delta \leq x / X \\
C_{M}+\left(C_{1}-C_{M}\right) x /(\delta X), \quad 0<x / X<\delta \\
C_{M}, \quad x=0 .
\end{array}\right.
$$


This initial condition causes packed beds to form in the batch case $V_{b}=$ 0 . Further, when characteristics enter $\Omega$ from the bottom of the thickener $x=0, t>0$, we assume boundary data of the form

$$
C(0, t)=C_{M}
$$

This circumstance might arise, for instance, when the outflow pumping speed $V_{b}$ is decreased as part of the control strategy. Similarly, when characteristics enter $\Omega$ from the top of the thickener $x=X, t>0$, we assume boundary data of the form

$$
C(1, t)=0 .
$$

The solution $C(x, t)$ of (5) can possess discontinuities whose properties depend on the precise nature of $V_{b}(t), V_{i}(t)$ and $C_{i}(t)$. In particular, a discontinuity sometimes propagates upwards from $x=0$, whilst other discontinuities propagate upwards and downwards from the injection zone near $x=h_{i} X$. Figure 3 illustrates a typical case. Under certain circumstances, the introduction of boundary condition (8) (necessitated by decreasing $V_{b}$ ) causes a discontinuity to form at the boundary $x=0$.

We are now able to state our fundamental goal:

Given constants $H^{*}, \varepsilon>0$ and the initial concentration distribution $f_{0}(x)$, determine the control variables $V_{b}(t)$ and $V_{i}(t)$ so that an interface exists between free settling particles and a high concentration zone. This interface is to be confined within an $\varepsilon$-neighbourhood of the height $x / X=H^{*}$.

If the above goal can be achieved, a second goal is

Characterise the concentration $C(0, t)$ of material removed at the bottom of the thickener.

\section{Analytical considerations as to the feasibility of control}

This paper considers a simple analytical model for a continuous thickener-the principal assumptions include monodisperse particle distribution, the empirical law (2) for $v_{s}(C)$, assumption of one-dimensional behaviour, the form chosen for the initial concentration $C(x, 0)$, neglect of particle momentum effects, and a simple treatment of the packed bed by virtue of 
the second term in (2). Despite these assumptions, the control problem is technically complicated and many cases need to be considered. An effective control strategy is deduced later in this section. First, however, we investigate the nature of the flux curve (3) for $\Phi(C)$ and discuss the general nature of the solution $C(x, t)$.

\subsection{Nature of the flux curves}

Consider $\Phi(C)$ below the feed at $x / X=h_{i}$ :

$$
\begin{aligned}
\Phi(C) & =V_{b} C+\phi(C) \\
& =C\left[V_{b}+v_{0}\left(1-C / C_{M}\right)^{q}+v_{1} C\left(C_{M}-C\right)\right]
\end{aligned}
$$

Here $\left[v_{0}, v_{1}, C_{M}, q\right]$ are parameters determined by the nature of the sedimenting system of particles and liquid, whilst $V_{b}$ is an adjustable parameter. For values of $\left[v_{0}, v_{1}, C_{M}, q\right]$ typical of those for common systems such as mud settling in water the curve $\Phi(C)$ has two inflection points $C_{p}^{1}, C_{p}^{2}$ with $0<C_{p}^{1}<C_{p}^{2}$. These points are independent of the value of $V_{b}$.

As $V_{b}$ increases, $\Phi(C)$ passes through four configurations as shown in Figure 2. To anticipate later developments, control is possible only if the flux curve is of type I, II or III. To achieve control, flux curves which are initially in type IV are converted to one of the other types by changing $V_{b}$.

\subsection{Nature of solutions of $(5,6)$}

Again, consider the zone below the feed. The method of characteristics (see e.g. $[6,16])$ can be used to solve $(5,6)$ subject to initial data $C(x, 0)=f_{0}(x)$. One case out of the four shown in Figure 2 is now analysed.

Suppose $\Phi(C)$ has type III behaviour and the injected concentration $C_{i}$ is such that there is an imbalance between the injected flux $V_{i} C_{1}$ and the flux below the feed $\Phi\left(C_{1}\right)$ as shown in Figure 3 (see p. 276) in which $V_{i} C_{i}>\Phi\left(C_{1}\right)$. Suppose also that $C_{1} \leq C_{\max }\left(V_{b}\right)$ where $\Phi^{\prime}\left(C_{\max }\left(V_{b}\right)\right)=0$. At the bottom of the thickener, a high concentration zone starts to build up because of the form chosen for the initial data $f_{0}(x)$. The top of this zone is the interface we eventually seek to control. In mathematical terms, the high concentration zone is formed by the intersection of characteristics emanating from the boundary segment $0<x / X<h_{i}, t=0$ thereby causing a primary discontinuity (or "shock") to form and move upwards through the thickener. The upward speed of this discontinuity is (Carrier \& Pearson [5, p. 232])

$$
W=-\frac{\Phi^{+}-\Phi^{-}}{C^{+}-C^{-}}
$$

where $+(-)$ denote values immediately above (below) the discontinuity. That is, the negative slope of the line $A C$ gives the initial upward speed of the top of the high concentration zone. 
In cases where $V_{i}<v_{s}+V_{b}$, conservation of sedimenting particles shows that the flux just below the feed is $\Phi\left(C_{i}^{f}\right)=V_{i} C_{i}$ (the point labelled $B$ in Figure 3). Characteristics emanating from $\delta<x / X<h_{i}, t=0$ intersect with those passing through $x / X=h_{i}, t>0$ and a secondary discontinuity moves downward through the thickener at speed given by the slope of $A B$. When the primary and secondary discontinuities indicated by $A B$ and $A C$ in Figure 3a meet, a tertiary discontinuity forms and moves upwards at speed given by the negative slope $B C^{\prime}$. In the absence of control adjustments, this discontinuity will move upwards through the whole thickener thus giving overload. The motion of the discontinuities is illustrated in Figure 3. We seek to control the location of the tertiary discontinuity.

Other cases can be similarly analysed using the method of characteristics. It is clear that a wide variety of cases can occur, particularly overload cases as described above and underload cases in which the high concentration zone at the bottom of the thickener is pumped away. Discontinuities can also rise

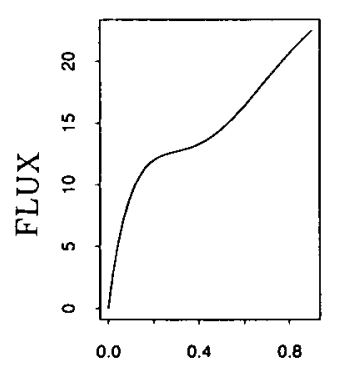

CONCENTRATION

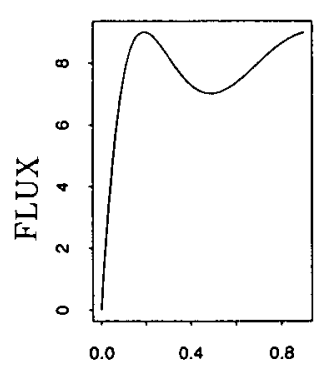

CONCENTRATION

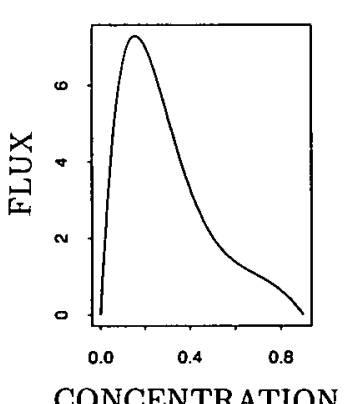

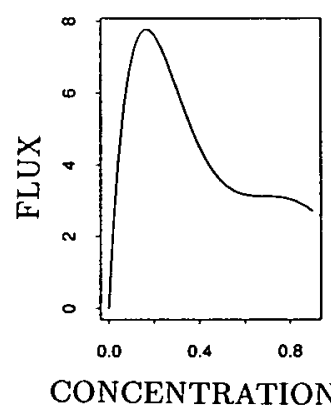

CONCENTRATION

FIGURE 2. Illustrating typical behaviour of $\Phi(C)$ for fixed $\left[v_{0}, v_{1}, C_{M}, q\right]$ and increasing $V_{b}$ : bottom right-type I, bottom left-type II, top right-type III, top left-type IV. 

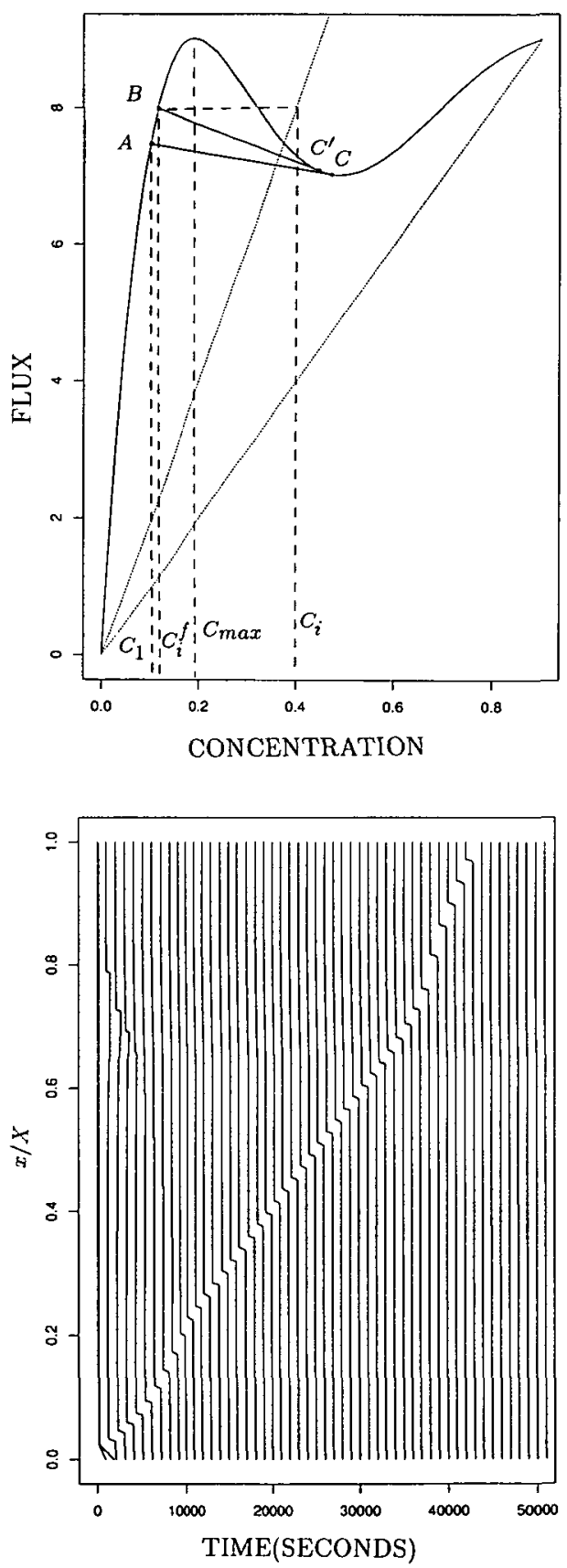

Figure 3. An overload case in the absence of control. The dotted lines are the equations $\Phi=V_{i} C_{i}$ and $\Phi=V_{b} C_{b}$. A primary discontinuity $A C$ builds up at the base of the thickener and eventually collides with the secondary discontinuity $A B$ dropping from the feed level $x=$ $h_{i}$. The resulting tertiary discontinuity $B C^{\prime}$ rises through the thickener. The right-hand figure shows a numerical simulation of the motion of discontinuities (see Section 4 ). 
from the top of the feed zone $x=h_{i}$, although this topic is not pursued here.

\subsection{Strategy for control}

The speed (10) of the tertiary discontinuity can be controlled by adjusting $\Phi^{-}$(by adjusting $V_{b}$ ) whilst maintaining $\Phi^{+}$constant (by a simultaneous adjustment of $V_{i}$ ). The essence of the control strategy is therefore to adjust the original value of $V_{b}$ (say $V_{b}^{0}$ ) by an amount $\Delta V_{b}$ so as to make the discontinuity stationary $(W=0)$. We have

$$
\begin{aligned}
W^{0} & =\frac{v_{s}\left(C^{+}\right) C^{+}-V_{b}^{0} C^{+}-v_{s}\left(C^{-}\right) C^{-}-V_{b}^{0} C^{-}}{C^{-}-C^{+}} \\
& =\frac{v_{s}\left(C^{+}\right) C^{+}-v_{s}\left(C^{-}\right) C^{-}}{C^{-}-C^{+}}-V_{b}^{0},
\end{aligned}
$$

where the superscript 0 denotes the initial value of a parameter such as $V_{b}$.

We now replace $V_{b}^{0}$ by $V_{b}^{0}+\Delta V_{b}$ and set $W$ to zero so that

$$
0=\frac{v_{s}\left(C^{+}\right) C^{+}-v_{s}\left(C^{-}\right) C^{-}}{C^{-}-C^{+}}-\left(V_{b}^{0}+\Delta V_{b}\right)
$$

that is

$$
\Delta V_{b}=W^{0} \text {. }
$$

If $C^{+}$and $C^{-}$are such that the case $W^{0}<-V_{b}^{0}$ occurs, we halve $V_{b}$ and impose the Dirichlet condition (8) at $x=0$. In practice, (8) is applied in all cases when $V_{b}$ is reduced: this can either lead to the growth of a new discontinuity at the bottom (if characteristics enter $\Omega$ at $x=0$ ) or, alternatively, the value $C=C_{M}$ simply propagates out the bottom if characteristics leave $\Omega$ at $x=0$.

Adjustments to $V_{b}$ produce a change in the particle flux below the feed and will lead to additional discontinuities propagating downward from the feed unless commensurate adjustments are made to the injection flux. It is simple to show the required adjustment to $V_{i}$ is

$$
\Delta V_{i}=C^{+} \Delta V_{b} / C_{i}
$$

for cases in which the injected concentration is held constant at $C_{i}$. For numerical implementation of the control strategy, we use a modification of (11); namely apply the adjustment $\Delta V_{b}=W^{0} \cdot(1+d), d \ll 1$ at $x / X=$ $H^{*} \cdot(1 \pm \varepsilon)$ after the discontinuity has passed through $x / X=H^{*}$. This has the effect of sending the discontinuity at reduced speed in the reverse direction towards the desired level $x / X=H^{*}$.

This control strategy is immediately applicable for rising discontinuities $\left(W^{0}>0\right)$ below the desired height $x / X=H^{*}$. If the rising discontinuity is 
above the desired height $x / X=H^{*}$, we could have made the discontinuity fall at the same speed by setting $\Delta V_{b}=2 W^{0}$. We would then have a falling discontinuity above the desired height $x / X=H^{*}$, and the discontinuity may be controlled by applying (11) at the appropriate time. In fact, however, the numerical implementation mentioned after (12) automatically controlled this case as well.

The case of a falling discontinuity below the desired height $x / X=H^{*}$ needs special attention. If the discontinuity's direction of motion is reversed by setting $\Delta V_{b}=2 W^{0}$, then characteristics might enter the region $\Omega$ through the bottom $x=0, t>0$ and boundary condition (8) is required. In this case, the concentration $C=C_{M}$ is carried into $\Omega$ by characteristics, and a close packed bed of particles will build up and can be controlled by applying strategy $(11,12)$ at $x / X=H^{*}$.

When the location of the tertiary discontinuity has eventually been controlled by the strategy above, the concentration produced at the bottom will have two possible values: the close packed value $C_{M}$ or the value $C_{m}\left(V_{b}\right)$ which locally minimises $\Phi(C)$. Which of these two cases is actually obtained depends on the initial concentration value $C_{1}$, the injected flux $V_{i} C_{i}$ (and hence the value $C_{i}^{f}$ ) and the nature of the $v_{s}(C)$ curve. An enumeration of all possible cases is not worthwhile; rather we point out the possibility of analysing any particular case using the method of characteristics (Section 3.2) or by direct numerical simulation (next section).

For economic operation of the thickener, all particles injected at the feed $x / X=h_{i}$ should drop down through the thickener eventually to be removed at the bottom. This condition holds provided

$$
V_{i} C_{i} \leq \Phi\left(C_{\max }\right)
$$

where $\Phi^{\prime}\left(C_{\max }\right)=0, \Phi^{\prime \prime}\left(C_{\max }\right)<0$.

\section{Numerical methods}

The sedimentation process is mathematically described in this model by (5) - a first order, quasi-linear pde. The solution of (5) was obtained using finite-difference schemes sensitive to transport errors. These schemes are easy to implement, and allow the tracking of multiple discontinuities and the possibility of generalisation to multi-dimensional and multi-component modelling. Full details of the numerical methods are given by Spencer [17].

A simple numerical scheme for (5) is the following explicit method which 
is first order accurate in space and time:

$$
C_{j}^{n+1}=C_{j}^{n}-\frac{\Phi_{j+1 / 2}^{n}-\Phi_{j-1 / 2}^{n}}{\Delta x_{j}}+V_{i} C_{i} K\left(x_{j} ; h_{i}, w\right) \Delta t
$$

where $\Phi_{j+1 / 2}$ is the flux sediment from the upwind direction past the $j+1 / 2$ grid position in time interval $\Delta t$ such that

$$
\Phi_{j+1 / 2}^{n}=\text { upwind }\left[C_{s}\left(v_{s}+V_{b}-V_{i} L\left(x_{j} ; h_{i}, w\right)\right]_{j+1 / 2}^{n} \Delta t\right.
$$

with a corresponding definition for $\Phi_{j-1 / 2}$.

The term $K\left(x_{j} ; p h_{i}, w\right)$ is the finite-difference representation of the Dirac delta function $\delta\left(x-h_{i}\right)$ denoting the feed level, over several grid blocks of total width $w$. The discretised form of the Heaviside function $H\left(x-h_{i} X\right)$ is the term $L\left(x_{j} ; h_{i}, w\right)$.

Upwind differencing guarantees monotonicity around shocks but introduces numerical diffusion that might degrade the accuracy of the solution. This problem can be alleviated by introducing a less diffusive higher-order scheme such as the Lax-Wendroff two-step method:

- Lax method diffusive half time step:

$$
C_{j+1 / 2}^{n+1 / 2}=\frac{1}{2}\left(C_{j+1}^{n}+C_{j}^{n}\right)-\frac{1}{\Delta x_{j+1 / 2}}\left(\Phi_{j+1}^{n}-\Phi_{j}^{n}\right)+\frac{\Delta t}{2} V_{i} C_{i} K\left(x_{j+1 / 2} ; h_{i} w\right)
$$

where the flux $\Phi^{n}$ acting over time interval $\Delta t / 2$ is

$$
\Phi_{j}^{n}=\left[\left(v_{s_{j}}+V_{b}-V_{i} L\left(x_{j} ; h_{i} ; w\right) C_{j}\right]^{n} \Delta t / 2\right.
$$

and the flux $\Phi_{j \pm 1 / 2}^{n+1 / 2}$ acting over the entire time interval $\Delta t$ is

$$
\Phi_{j \pm 1 / 2}^{n+1 / 2}=\left[\left(v_{s_{j \pm 1 / 2}}+V_{j \pm 1 / 2}\right) C_{j \pm 1 / 2}\right]^{n+1 / 2} \Delta t
$$

- Forward in time, centered in space (FTCS) full time step solution:

$$
C_{j}^{n+1}=C_{j}^{n}-\frac{\left(\Phi_{j+1 / 2}^{n+1 / 2}-j \Phi_{j-1 / 2}^{n+1 / 2}\right)}{\Delta x_{j}}+\Delta t V_{i} C_{i} K\left(x_{j} ; h_{i}, w\right)
$$

Unfortunately, higher-order schemes such as the Lax-Wendroff two-step method introduce dispersion errors that are dominant near discontinuities. These are manifested as 'ripples' that grow over time and destroy the solution. It is desirable to couple the improved accuracy of higher-order schemes with the guaranteed monotonicity of lower-order schemes near discontinuities. One technique that achieves this synthesis is flux corrected transport (FCT) introduced by Boris and Book [4] and Zalesak [20].

FCT determines the flux $\Phi$ at any time step as a weighted average of the flux produced by a low-order and a higher-order scheme. The potentially 
more accurate higher-order flux is used as much as possible without introducing the 'ripple' effect (flux correction). Basic steps of the general procedure as given by Zalesak are outlined below.

1. Determine $\Phi_{j \pm 1 / 2}^{L}$, the low-order scheme flux for a locally monotonic but diffused solution (across any particular discontinuity).

2. Determine $\Phi_{j \pm 1 / 2}^{H}$, the higher-order scheme flux.

3. Determine the new time step low-order solution.

4. Find the "anti-diffusive flux"

$$
A_{j \pm 1 / 2}=\Phi_{j \pm 1 / 2}^{H}-\Phi_{j \pm 1 / 2}^{L} .
$$

5. Correct the anti-diffusive flux so that nonphysical extrema are not introduced into the solution (extrema not found in the lower-order solution)

$$
A_{j \pm 1 / 2}^{C}=\lambda_{j \pm 1 / 2} A_{j \pm 1 / 2}
$$

such that

$$
0 \leq \lambda_{j+1 / 2} \leq 1
$$

6. Use the corrected anti-diffusive fluxes to compute the final updated solution

$$
C_{j}^{n+1}=C_{j}^{n+1, L}-\left(A_{j+1 / 2}^{C}-A_{j-1 / 2}^{C}\right) / \Delta x_{j} .
$$

7. Determine flux correction factors $\lambda_{j+1 / 2}$.

Algorithms for determination of the corrected anti-diffusive flux (21) are given $[4,20]$. The Zalesak formalism was used in our work.

First-order upwind differencing was chosen as the lower-order scheme for the FCT method. The quasi-linear nature of (5) means that the speed of characteristics defined by

$$
\Gamma=\gamma+V_{b}-V_{i} H\left(x-h_{i} X\right)
$$

is concentration and bulk velocity dependent. Therefore $\Gamma$ might take opposing signs in adjacent grid blocks-a situation most likely to occur across discontinuities. Hence the sign of the characteristic velocity in any given grid block is not always an accurate indicator of the upwind direction. This possibility has been taken into account in the current formulation-see Spencer [17] for details. Another consequence of the quasi-linear nature of (5) is the possibility of tighter restrictions on time step/grid block size than suggested by the usual Courant-Friedrichs-Levy condition for an explicitly upwind discretised linear hyperbolic equation. A further restriction on time step/grid block size to maintain numerical stability might occur due to the flux of injected material entering grid blocks at the feed level. 
The two-step Lax-Wendroff scheme was used as the higher-order method for Stage 2 of the FCT approach. Results by FCT using this method are considerably more accurate than by first-order upwind differencing aloneparticularly in producing sharp discontinuities. The explicit nature of the Lax-Wendroff scheme makes it computationally inexpensive, easy to implement and sufficiently accurate for our purposes. Our results were confirmed by tests using more accurate schemes such as those described by Morrow and Steinle $[14,18]$, Spencer [17].

\section{Results}

This section contains some cases to illustrate the control procedures described in the previous section. The following values were taken for parameters in expressions (2) for $v_{s}(C)$ and (7) for $f_{0}(x)$ :

$$
v_{0}=1.18 \times 10^{-4} \mathrm{~ms}^{-1}, \quad v_{1}=10^{-5} \mathrm{~ms}^{-1}, \quad q=5, \quad C_{M}=0.9, \quad C_{1}=0.1 .
$$

Figures 3-9 display the concentration $C(x, t)$ so that the zero of the concentration occurs at increasing values of the abscissa as time increases. This device enables the motion of the discontinuities to be clearly seen.

\section{Straightforward control}

Figure 4 (see p. 282) shows a simple case in which a rising tertiary discontinuity is controlled at a level $x / X=H^{*}$ which is above the level of intersection of the primary and secondary discontinuities. The output concentration $C(0, t)$ in this case is $C_{m}$ where $\Phi^{\prime}\left(C_{m}\right)=0, \Phi^{\prime \prime}\left(C_{m}\right)>0$. This case, in which the output concentration $C_{m}$ is less than the close-packed value $C_{M}$, might be less than optimal for practical operation of the thickener.

Tertiary discontinuity descending above $x / X=H^{*}$

Figure 5 (see p. 283) shows a simple case in which the tertiary discontinuity initially descends after it has been formed by the intersection of the primary and secondary discontinuities. The falling tertiary discontinuity is then controlled using (11) at $x / X=H^{*}$. The output concentration asymptotes to $C_{m}$.

Tertiary discontinuity descending and below $x / X=H^{*}$

Figure 6 (see p. 284) shows a case in which the tertiary discontinuity initially descends after it has been formed and the discontinuity is initially dropping faster than $V_{b}$. The output speed $V_{b}$ is then halved at each time step until the tertiary discontinuity starts to rise. Control at the desired height $x / X=H^{*}$ is achieved by increasing $V_{b}$ as in (11). The output concentration is the close-packed value $C_{M}$. 


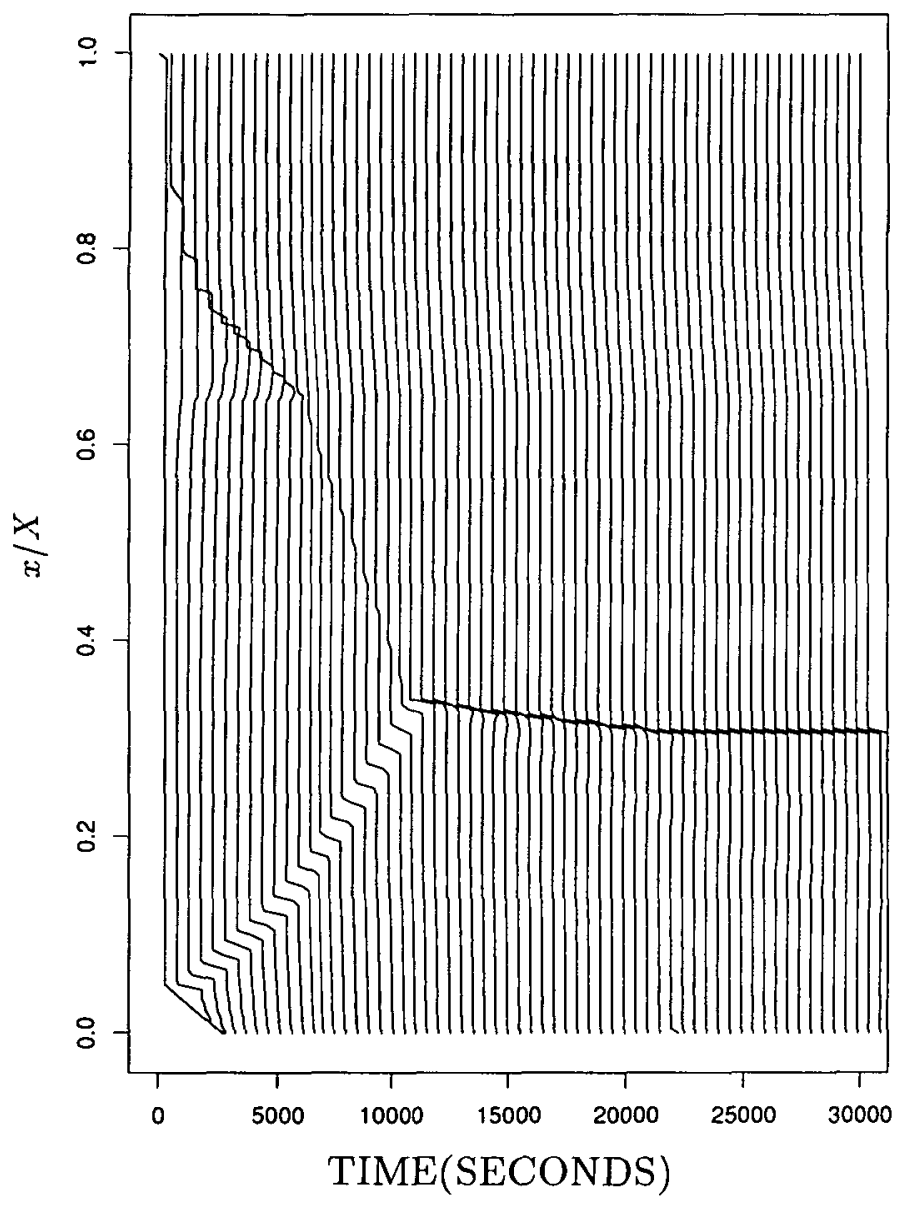

FIGURE 4. Illustration of control of a rising tertiary discontinuity initially below the desired control height $H^{*}=0.375 X$. The feed is between $0.65 X$ and $0.85 X$.

Two primary discontinuities; control of descending tertiary discontinuity

Figure 7 (see p. 285) shows a more complicated case in which the flux curve is initially of type II and there are two primary discontinuities. The tertiary discontinuity, which would otherwise rise after its formation, is made to fall by a single increase in $V_{b}$. This causes the primary discontinuity rising from $x=0$ to reverse direction and eventually disappear. Control at $x / X=H^{*}$ is then achieved by decreasing $V_{b}$ as in (11). The output concentration is $C_{m}$. Two primary discontinuities - a further case

Figure 8 (see p. 286) shows a case which is a variation on the previous case. Now the parameters are such that the primary discontinuity rising from $x=0$ does not reverse its direction of motion as $V_{b}$ is increased. As the 


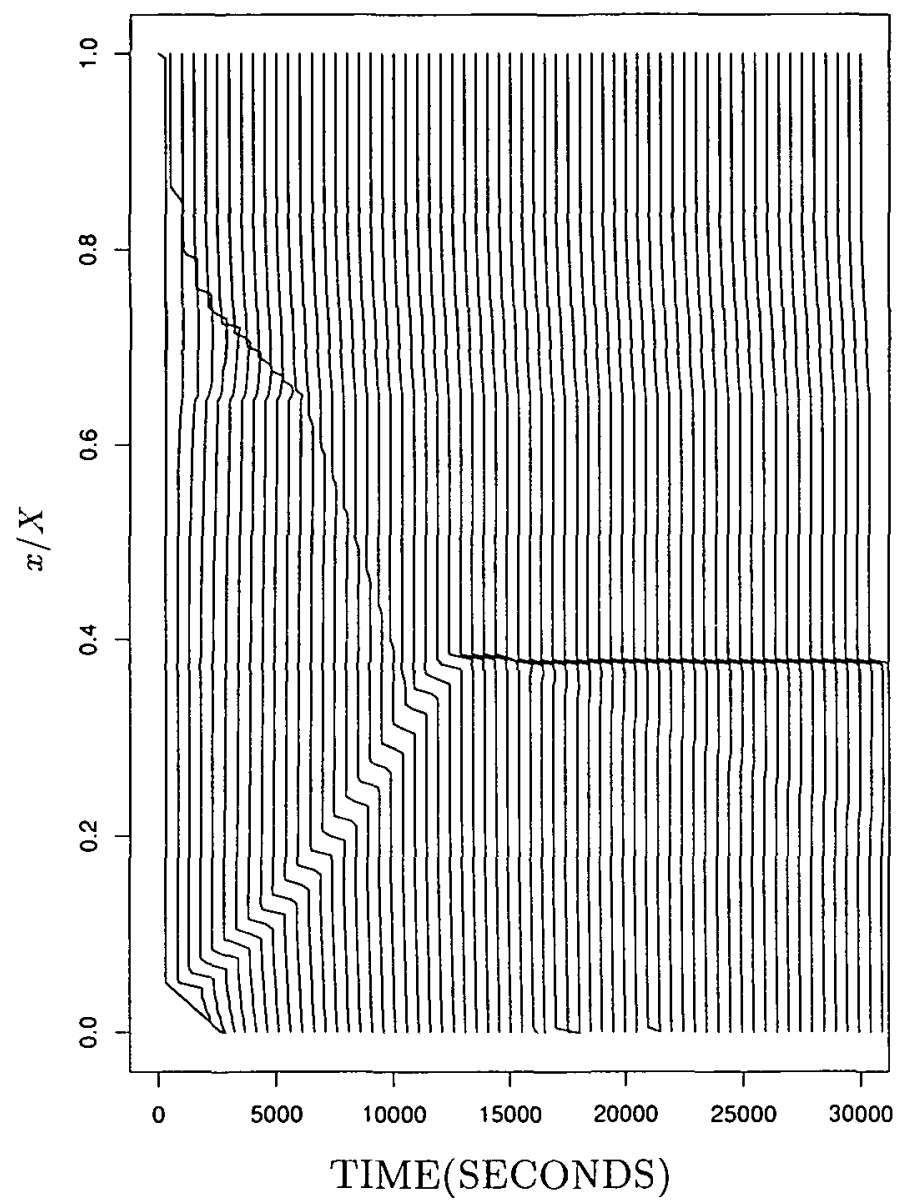

FIGURE 5. Illustration of control of a descending tertiary discontinuity initially above the desired control height $H^{*}=0.30 X$.

tertiary discontinuity drops towards $x / X=H^{*}, C^{-}$increases and continued reductions in $V_{b}$ are required. Eventually, the primary discontinuity rising from $x=0$ collides with the tertiary discontinuity and a final correction is made to achieve the steady state solution. The final output concentration is $C_{M}$.

\section{Two primary discontinuities-another case}

Figure 9 (see p. 287) shows a case in which the tertiary discontinuity would initially fall after its formation except for the fact that control has been applied immediately, causing the tertiary discontinuity to rise. During the application of control, boundary condition (8) was applied at $x=0$ causing 
another primary discontinuity to build up. The tertiary discontinuity is controlled at $x / X=H^{*}$; further adjustment (i.e. dynamic control) is required until the second primary discontinuity collides with the controlled-tertiary discontinuity. At this point, one last adjustment is made and the steady state is achieved. The final output concentration is $C_{M}$.

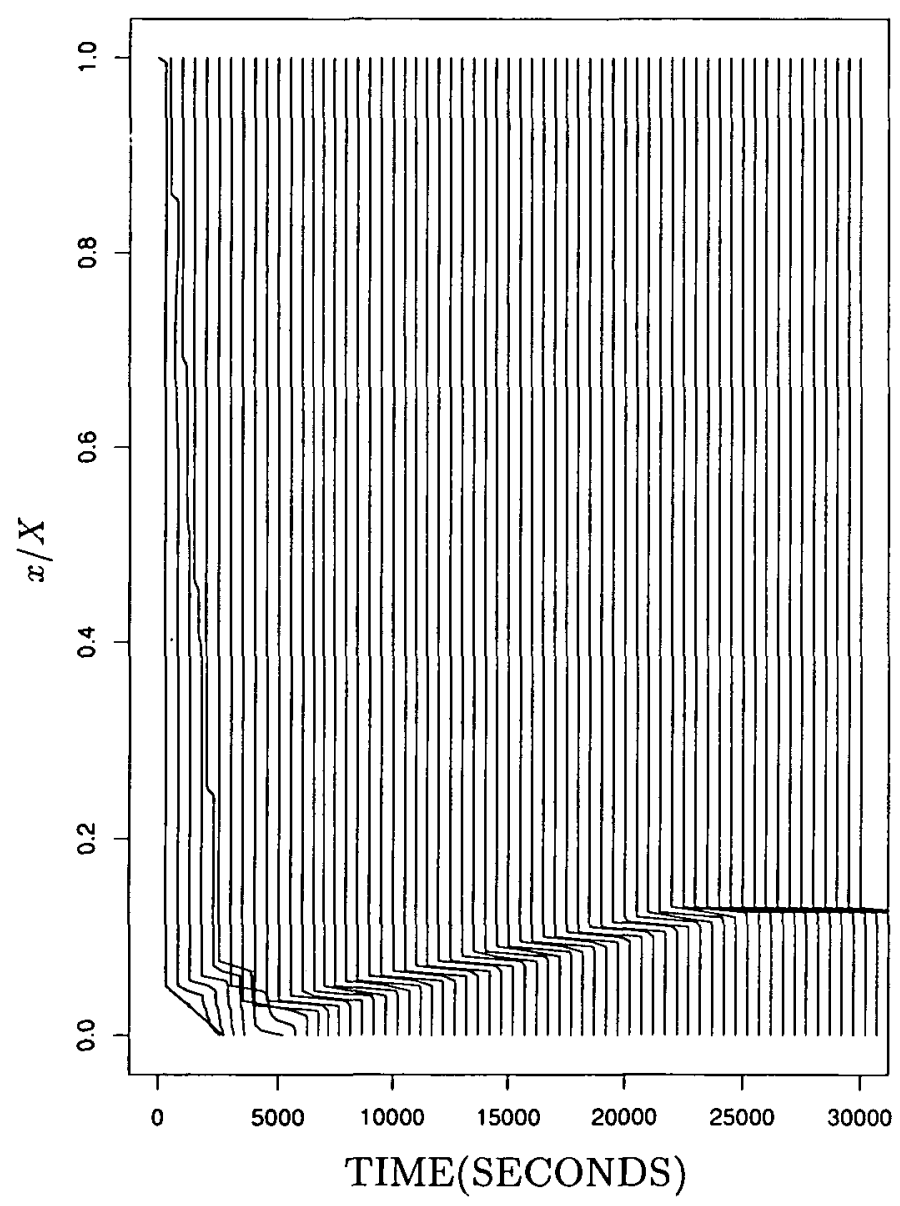

FIGURE 6. Illustration of control of a falling tertiary discontinuity initially below the desired control height $H^{*}=0.125 X$. 


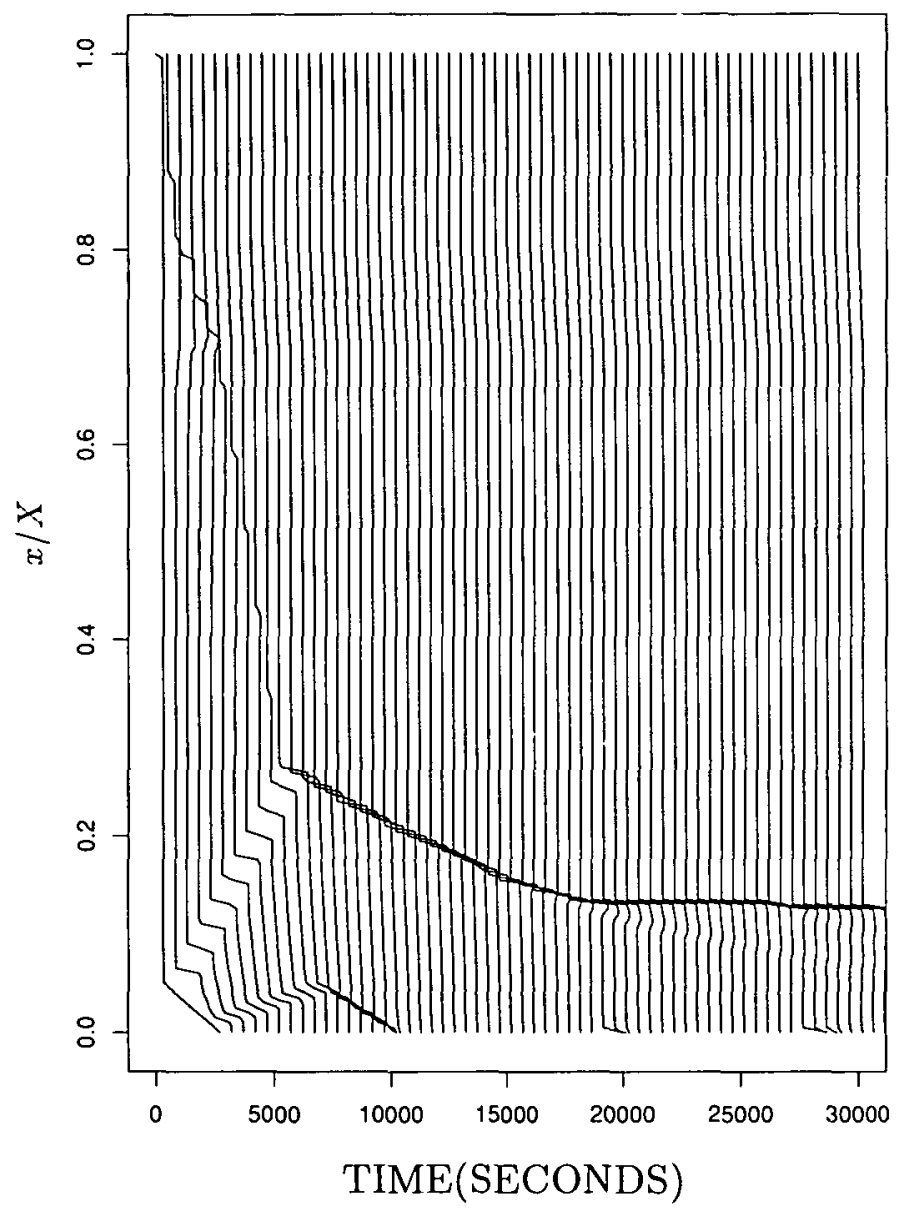

Figure 7. A situation like that of Figure 5 except that the parameter settings also lead to a close-packed bed rising from $x=0$. This bed disappears when control is applied. 


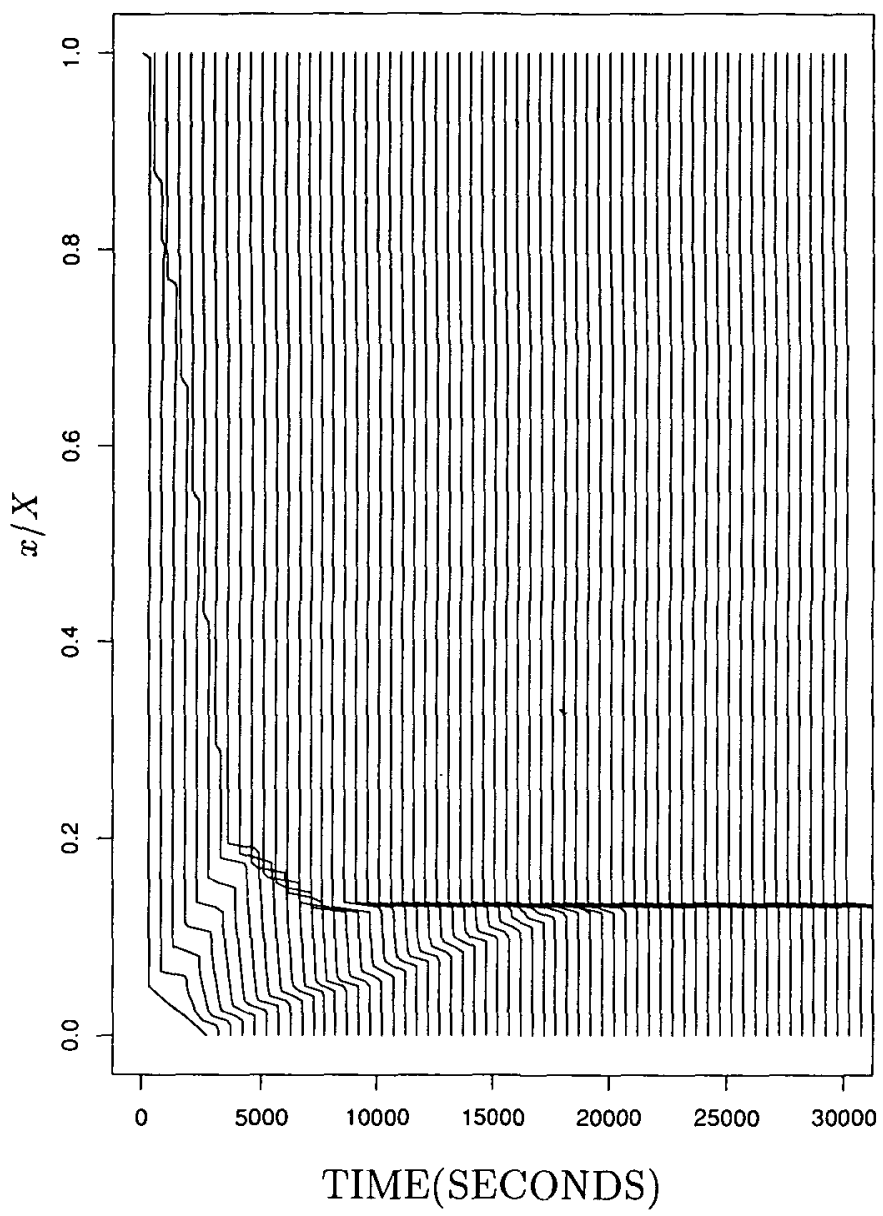

FIGURE 8. A situation like that of Figure 7 except that the initial parameter settings are such that the close-packed bed continues to rise after control is applied. Subsequent control of the close-packed bed is required (see description in text). 


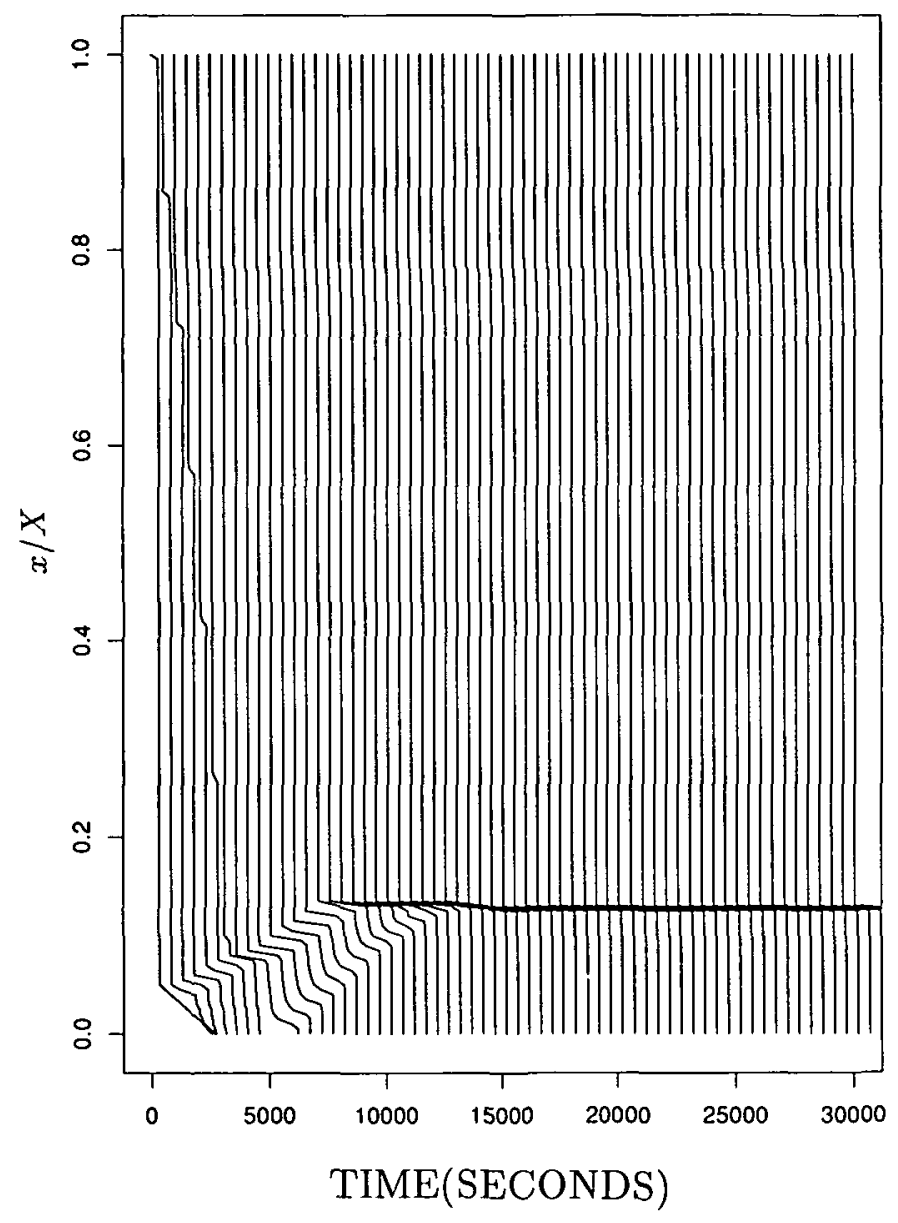

Figure 9. A situation like that of Figure 6 except that a separate primary discontinuity forms and must be controlled (see description in text). 


\section{Conclusions}

A control strategy based on a judicious use of the pumping speeds $V_{b}$ and $V_{i}$ has been suggested in this paper; the feasibility of this strategy has been confirmed numerically. The basis for the numerical work was code which had previously been written to simulate the performance of continuous thickeners. More accurate algorithms were used during the development stage to confirm the accuracy of the results.

The model problem considered in this paper was based on stringent simplifications mentioned in Sections 1 to 3. It is clear that the fine details of the control process depend sensitively on the initial boundary conditions and the nature of the flux curve $\Phi(C)$ in which is incorporated the empirical $v_{s}(C)$ relationship. It was found that there are many possible cases that can be considered, and we have given illustrative examples for some of these cases.

Events taking place above the feed level have not been considered in detail here. In fact, we have used parameter settings which ensure the discontinuities do not rise up from the feed level. Such cases might be of economic significance, in which case they deserve further investigation.

Another aspect which might be of economic significance is control of the output concentration $C(0, t)$. We believe that it is possible to control both $C(0, t)$ and the discontinuity height $x / X=H^{*}$, although this might require assumption of variability in the injection concentration $C_{i}(t)$ as well as the input and output pumping speeds $V_{i}$ and $V_{b}$.

\section{References}

[1] F. M. Auzerais et al., "The resolution of shocks and the effects of compressible sediments in transient settling”, J. Fluid Mech. 195 (1980) 437-462.

[2] N. G. Barton et al., "Sedimentation in concentrated suspensions", Xih Int. Congress of Rheology, Sydney (1988) 173-176.

[3] N. G. Barton et al., "Experimental and theoretical studies of the sedimentation of concentrated suspensions", Proc. Sth Australasian Conf. on Heat and Mass Transfer, Christchurch, (1989).

[4] J. P. Boris and D. L. Book, "Flux-corrected transport. III. Minimal-error F.C.T. algorithms", J. Comp. Physics 20 (1976) 397-431.

[5] G. F. Carrier and C. E. Pearson, Partial differential equations. Theory and technique, (Academic Press, New York, 1976).

[6] R. Courant and D. Hilbert, Methods of mathematical physics Vol. II, (J. Wiley, New York, 1962).

[7] D. C. Dixon, “Momentum-balance aspects of free-settling theory. I. Batch thickening”, Separation Science 12 (1977) 171-191.

[8] B. Fitch, "Batch tests predict thickener performance", Chemical Engineering August 23, (1971) 83-88. 
[9] B. Fitch, “Kynch theory and compression zones", AIChE J. 29 (1983) 940-947.

[10] C. Fryer and P. H. T. Uhlherr, "Continuous thickeners-behaviour and design", Eighth Aust. Chem. Eng. Conf., Melbourne (1980) 80-84.

[11] I. Howells et al., "Time-dependent batch settling of flocculated suspensions", Appl. Math. Modelling Vol. 14 (1990) 77-86.

[12] G. J. Kynch, “A theory of sedimentation” Trans. Faraday Soc. 48 (1952) 166-176.

[13] K. A. Landman, L. R. White and R. Buscall, "The continuous-flow gravity thickener. steady state behavior", AIChE J. 34 (1988) 239-252.

[14] R. Morrow and P. Steinle, "Implicit flux-corrected transport" in Computational Techniques and Applications: CTAC-87, (eds. J. Noye and C. Fletcher), (Hemisphere Publishing, New York, 1988) 499-508.

[15] K. H. Park, J. R. G. Andrews and P. H. T. Uhlherr, "Batch sedimentation and thickener behaviour", Proc. 3rd Pacific Chem. Eng. Congress Vol. 1 (1983) 100-105.

[16] S. J. Spencer, D. R. Jenkins and N. G. Barton, "Modelling of batch and continuous sedimentation of suspensions", in Computational Techniques and Applications: CTAC89 (eds. W. L. Hogarth and B. J. Noye), (Hemisphere, New York, 1989) 501-508.

[17] S. J. Spencer, "Modelling of continuous sedimentation of suspensions”, Technical Report NSW 89/22 (CSIRO Division of Mathematics and Statistics, Sydney, 1990).

[18] P. Steinle and R. Morrow, "An implicit flux-corrected transport algorithm", J. Comp. Physics 80 (1989) 61-71.

[19] F. M. Tiller, “Revision of Kynch sedimentation theory", AIChE J. 27 (1981) 823-829.

[20] S. T. Zalesak, "Fully multidimensional flux-corrected transport algorithms for fluids", J. Comp. Physics 31 (1979) 335-362. 